\title{
Identificación del fenotipo de inestabilidad microsatelital en carcinoma colorrectal mediante el análisis de la expresión de proteínas reparadoras del ADN: Revisión narrativa
}

\author{
Identification of microsatellite instability phenotype in colorectal carcinoma through \\ expression analysis of DNA mismatch repair proteins: Narrative review \\ Orlando Rodas Pernillo (iD *1,2, Edith Oregón*3
}

${ }^{1}$ Doctorado en Ciencias Biomédicas, Escuela de Estudios de Postgrado, Facultad de Ciencias Médicas, Universidad de San Carlos ${ }^{2}$ Departamento de Laboratorios Clínicos, Sección de Patología, Hospital Roosevelt, ${ }^{3}$ Doctorado en Ciencias Biomédicas, Centro Universitario de las Ciencias de la Salud, Universidad de Guadalajara, México

*Autor al que se dirige la correspondencia: kescobarcastro@gmail.com

Recibido: 26 de junio 2020 / Revisión: 23 de marzo 2021 / Aceptado: 06 de julio 2021

\section{Resumen}

$\mathrm{E}$ 1 carcinoma colorrectal (CCR) es de las primeras causas de mortalidad del mundo, presentando Guatemala una incidencia anual de 7.4/millón de habitantes. El síndrome de Lynch se caracteriza clínicamente por un inicio temprano del CCR con lesiones causadas por alteraciones en genes que codifican proteínas reparadoras. Los microsatélites son regiones del ADN con una unidad repetitiva de uno o más nucleótidos y son susceptibles a errores durante la replicación de ADN de los enterocitos. Existe un sistema de reparación que corrige estos errores. Cuando las proteínas reparadoras de este sistema están mutadas o ausentes, dichos errores del ADN persisten. Estas proteínas reparadoras se expresan en el núcleo de las células colónicas normales y son detectables utilizando estudios de inmunohistoquímica (IHQ). Los genes $M L H 1$ y MSH2 pueden encontrarse mutados en el $90 \%$ de los casos de cáncer colorrectal y el resto corresponde a MSH6 y PMS2. Esta vía oncogénica se caracteriza por alteración del sistema de reparación de errores durante la replicación del ADN, controlado por los genes MMR (mismatch repair), principalmente $M L H 1, M S H 2$, MSH6 y PMS2. Se realizó una revisión extensa de la literatura en PubMed, Springer y JAMA, usando las palabras clave: fenotipo de CCR, Síndrome de Lynch e inestabilidad microsatelital, detectándose 55 artículos. El objetivo de esta revisión es describir la importancia de la identificación del fenotipo del CCR por medios de IHQ y de pruebas moleculares para el eficaz tratamiento con inmunoterapia anti-PD1/PD-L1.

Keywords: Cáncer de Colon; MLH1; MSH2; MSH6; PMS2; Síndrome de Lynch

Abstract

Colorectal cancer (CRC) is one of the leading causes of mortality in the world. In Guatemala it's an important cause of morbidity (7.4 per million inhabitants). Lynch syndrome is clinically characterized by an early onset of nonpolyposis colorectal carcinoma, with multiple lesions and neoplasms. The syndrome is caused by mutations in genes encoding DNA mismatch repair proteins. The microsatellites are regions of the DNA that repeat between one or more nucleotides and are susceptible to errors during replication, these are corrected by a repair system, when genes are mutated, the errors persist. The genes encoding repair proteins are expressed in the nuclei of normal colonic cells which can be observed using immunohistochemical studies. The MLH1, MSH2 genes are found to be mutated in $90 \%$ of the cases and the rest corresponds to the MSH6 and PMS 2 genes. This oncogenic pathway characteristically consists of an alteration in the DNA repair system that is controlled by mismatch repair genes (MMR). An extensive research was conducted on PubMed, Springer and JAMA, using the keyword: CRC phenotype, Lynch syndrome and microsatellite instability. 55 articles were found. . This review«s objective is to understand the mechanisms of nonpolyposis colorectal cancer and the importance of identifying patients with a mutant phenotype as a predictive factor for the efficacy of the anti-PD1/PDL1 immunotherapy and for prognosis.

Palabras claves: Colon Cancer; $M L H 1 ;$ MSH2; MSH6; PMS2 


\section{Introducción}

La incidencia del Carcinoma Colorrectal (CCR) varía en función de la edad, apreciándose un incremento notorio en las tasas de incidencia a partir de los 50 años (Burbano Luna et al., 2016). La supervivencia del CCR ha mejorado en los últimos años. En España, la supervivencia a los 5 años de diagnosticado el CCR se sitúa dentro de la media de supervivencia de los países europeos que es de $49.5 \%$ para cáncer de colon y $43 \%$ para cáncer de recto). Esta supervivencia depende fundamentalmente del estadio tumoral en el momento del diagnóstico. La etiología del CCR es heterogénea (Deen et al., 2016; Navarro et al., 2012).

Se han descrito factores ambientales y genéticos relacionados con la aparición del CCR (Bumbano Luna et al, 2016). Los estudios genéticos tienen alta sensibilidad y especificidad y son una opción válida para la detección del fenotipo mutador de síndrome de Lynch que es un desorden autosómico dominante caracterizado clínicamente por un inicio temprano del CCR con lesiones múltiples y neoplasias de otras localizaciones como endometrio, estómago, intestino delgado, riñón, ovario y piel y está causado por alteraciones en los genes que originan las proteínas reparadoras. Aunque la historia familiar y las características clínicas pueden sugerir cáncer hereditario no polipósico, la identificación del factor de riesgo hereditario no se realiza en un gran número de familias. Por lo anterior, se recomienda el estudio de inestabilidad microsatelital de rutina, mediante la técnica de IHQ que ha demostrado ser altamente sensible para la detección de inestabilidad microsatelital, y que, además, es más económico que los estudios moleculares ya que busca la pérdida de expresión de las proteínas MLH1, MSH2, MSH6 y PMS2 (Burbano Luna et al, 2016).

El tamizaje mediante IHQ puede reducir la mortalidad en pacientes con diferentes fenotipos de inestabilidad de microsatélites, lográndose identificar el síndrome de Lynch tras el estudio germinal. Además se pueden detectar diferentes fenotipos de inestabilidad de microsatélites diferentes a Síndrome de Lynch, dado que algunos fenotipos pueden presentar respuesta frente a medicamentos específicos como el 5ñfluorouracilo (Burbano Luna et al., 2016). Adicionalmente, puede reducir su incidencia mediante la prevención de nuevas ocurrencias ya que se pueden detectar tempranamente pólipos pre cancerosos (Deen et al., 2016) y favorecer el seguimiento de casos presintomáticos en aquellos portadores de alteración genética mediante los estudios familiares.
El objetivo de esta revisión es describir la importancia de la identificación del fenotipo del CCR por medios de IHQ y pruebas moleculares para su tratamiento y evaluación pronóstica. Para ello, se realizó una revisión extensa de la literatura en las bases de datos de PubMed, Springer, Scopus y JAMA, usándose como criterios de inclusión el estudio del fenotipo de CCR y detectándose 55 artículos no mayores a 5 años de búsqueda (a los cuales se tuvo acceso gratuito o pagado a 47 de ellos) que fueron revisados y analizados en esta revisión.

\section{Contenido}

\section{Formas heredofamiliares y esporádicas de CCR}

Dentro de los cuadros heredables del CCR se encuentran dos formas: los pacientes con síndrome de Lynch o cáncer de colon hereditario no polipósico y los pacientes con síndrome polipósico. Se han descrito cinco formas heredofamiliares de CCR. Las cinco formas son las asociadas a enfermedad inflamatoria intestinal, poliposis adenomatosa familiar, la forma asociada al gen MUTYH (que sintetiza la MutY DNA Glycosylase), síndrome de Lynch y el CCR familiar. Con respecto a la patogénesis se observan tres mecanismos patogénicos; la inestabilidad microsatélite, inestabilidad cromosómica y el fenotipo metilador de islas CpG (Castells et al., 2009).

En la forma polipósica familiar, el defecto molecular es la vía APC/WNT con el gen diana $A P C$, de transmisión autosómica dominante, sin localización predominante para el CCR, con histología tubulovellosa y adenocarcinoma típico. La forma de poliposis adenomatosa familiar, causada por alteraciones en el gen MUTYH, con transmisión recesiva, histológicamente se manifiesta como adenoma aserrado sésil y adenocarcinomas mucinosos, sin localización predominante.

La forma del CCR hereditario no polipósico, con alteración en los genes de la reparación del ADN MSH2, MLH1, MSH6 y PMS2 y transmisión autosómica dominante, se localiza predominantemente en el colon derecho, presentando histología de adenoma serrado sésil y adenocarcinoma mucinoso (Battaglin et al., 2018).

El cáncer de colon esporádico, con defecto molecular Vía APC/WNT, sin transmisión hereditaria, es decir, alteraciones somáticas en el tejido afectado, que se localiza en el lado izquierdo, presenta histología 
tubular, vellosa y adenocarcinoma típico. El cáncer de colon esporádico, con reparación de errores en el apareamiento del ADN de genes MSH2, MLH1, sin transmisión, los cuales se localizan en el colon derecho y presentan histología de adenoma serrado sésil y adenocarcinoma mucinoso.

Se estableció una clasificación (en 2015 por Guinney et al.) en el cual destacan cuatro subtipos moleculares:

Consenso molecular subtipo 1 (CMS1), el cual mostró inestabilidad de microsatélites (IMS+), fenotipo hipermutado (MICpG-H) y mutaciones en $B R A F$.

El subtipo inmune de IMS (CMS2) se caracterizó por una expresión aumentada de genes asociados con infiltrados inmunes y fuerte activación de las vías de evasión inmune. Estos tumores se presentaron comúnmente en el lado izquierdo. Los pacientes tuvieron mejores tasas de supervivencia después de la recaída en comparación con CMS1.

Consenso molecular subtipo 3 (CMS3), la mayoría de estos tumores presentan una mezcla de inestabilidad cromosómica y de microsatélites, principalmente MICpG-L y tuvieron baja frecuencia de alteraciones en el número de copias. Las mutaciones en KRAS fueron comunes.

Consenso subtipo molecular 4 (CMS4), el 23\% de la cohorte clasificó en este subtipo mesenquimal, caracterizado por prominente actividad del factor de crecimiento transformante- $\beta$, invasión estromal y angiogénesis. Tuvo alta frecuencia de ganancias y pérdidas en el número de copias en distintos cromosomas. Los tumores tienden a ser diagnosticados en una etapa posterior y muestran la mayor recaída y la menor supervivencia global (Alhilfi et al., 2019).

\section{Patogénesis de CCR}

Las vías principales de carcinogénesis son inestabilidad cromosómica, inestabilidad de microsatélites y la vía serrada o fenotipo metilador. Este último se refiere a la modificación del ADN causadas por la metiltransferasa de $\mathrm{ADN}$ y resulta en la adición covalente del grupo metilo $\left(-\mathrm{CH}_{3}\right)$ al carbono 5 de la citosina de $\mathrm{CpG}$ en la región promotora del gen.

Con respecto a las alteraciones genéticas, la mayoría $(90 \%)$ de los CCR esporádicos presenta activación de determinados oncogenes (KRAS) e inhibición de genes supresores (DCC, APC, TP53). Los tumores originados por esta asociación de alteraciones, denominada vía supresora, muestran entre sus características presentar inestabilidad cromosómica. Los tumores con inestabilidad cromosómica presentan con frecuencia alteraciones del cariotipo, con ganancias y pérdidas cromosómicas, así como translocaciones. De la misma forma, las pérdidas alélicas son relativamente frecuentes, presentando un desequilibrio alélico a nivel de múltiples loci. El factor presumiblemente iniciador de esta vía sería la pérdida de un gen supresor como es el gen $A P C$.

Existe una segunda vía oncogénica caracterizada por la alteración del sistema de reparación de errores durante la replicación del ADN, controlado por los genes MMR, principalmente $M L H 1, M S H 2, M S H 6$ y $P M S 2$. Los tumores desarrollados por esta vía presentan alteraciones en el número de repeticiones de los microsatélites al comparar tejido tumoral con tejido sano del mismo paciente. Los tumores esporádicos MMR+ se originan debido a inactivación por hipermetilación del promotor del gen $M L H 1$. En estos casos siempre se observa pérdida de expresión inmunohistoquímica de MLH1 (Payá Romá et al., 2006; Perea et al., 2011).

Para denominarlos se han aplicado en la literatura diversos términos como tumores con inestabilidad de microsatélites (IMS+), tumores con alteración de la vía reparadora (MMR+), tumores con fenotipo RER+ (replication errors) o tumores con fenotipo mutador. Los tumores MMR+ presentan un mejor pronóstico que los tumores sin alteración de la vía reparadora. Además, no se benefician de los tratamientos quimioterápicos basados en terapias con 5-fluorouracilo. La gran mayoría de tumores con IMS (83\% aproximadamente) son esporádicos, el $17 \%$ restante se desarrolla en pacientes con CCR hereditario no polipósico (CCHNP) o síndrome de Lynch.

Se considera que los tumores con inestabilidad cromosómica y aquellos que presentan inestabilidad microsatelital serían mutuamente excluyentes (Payá Romá et al., 2006; Perea et al., 2011).

\section{Síndrome de Lynch en CCR}

En 1913 el patólogo Aldred Warthin informó sobre cuatro familias con antecedentes sorprendentes de cáncer multigeneracional que se determinaron a partir de una revisión de los registros médicos de los pacientes tratados en la Universidad de Michigan (Lynch et al., 2009). En 1966, Henry Lynch informó sobre dos familias numerosas de Nebraska y Michigan, con un espectro similar de cánceres colorrectales asociado a tumores de endometrio y de estómago, y los diagnosticó 
como "síndrome de cáncer familiar". Posteriormente, se cambió su nombre a CCR hereditario no asociado a poliposis, para diferenciarlo de otra entidad previamente conocida como poliposis adenomatosa familiar, la cual es un síndrome hereditario por mutación del gen $A P C$ (Lynch et al., 2009; Ortiz et al., 2016). En la actualidad, se utiliza el nombre de síndrome de Lynch solo cuando se trata de la manifestación clínica de la mutación genética confirmada de alguno de los genes reparadores del ADN o pérdida de expresión del gen $M S H 2$ por deleción en el gen EpCAM (Giardiello et al., 2014).

El síndrome de Lynch se caracteriza clínicamente con un inicio temprano de $\mathrm{CCR}$, con lesiones múltiples y neoplasias de otras localizaciones como endometrio, estómago, intestino delgado, riñón, ovario y piel. Tiene su origen en mutaciones de genes que codifican enzimas que reparan los errores del ADN en el proceso de replicación. Desde el punto de vista conceptual, hay tres estrategias posibles para identificar a los pacientes con este síndrome: (a) la utilización de criterios clínicos; (b) el empleo de técnicas moleculares como la inestabilidad de microsatélites (IMS) e IHQ; y, (c) la combinación de ambas. Uno de los principales retos en la práctica clínica es la identificación de los individuos portadores de alteración en los genes reparadores del ADN, con el fin de favorecer la prevención del CCR a través de oportunas medidas de consejo genético (Vasen et al., 2013).

\section{IHQ y patología molecular para el tamizaje de Síndrome de Lynch}

El enfoque para el tamizaje del síndrome de Lynch se basa en estudios de IHQ para determinar la ausencia de expresión de MLH1 y al resultar comprobada la metilación en forma indirecta por medio de IHQ, se realicen pruebas de patología molecular para comprobar la hipermetilación que desencadenó la ausencia de expresión de MLH1. Este es el enfoque actual, sin embargo, un estudio basado en 1011 casos de CCR fueron tamizados para enfermedad de Lynch tanto con $M L H 1$ por IHQ y para $B R A F$ y se determinó que un tamizaje para $M L H 1$ por IHQ fue suficiente para disminuir la necesidad de realizar estudios de patología molecular en 12.7\% (Adar et al., 2017).

Los estudios de inmunohistoquímica deben realizarse sobre tejido fijado en formol al $10 \%$ e incluido en parafina. Para su interpretación diagnóstica deben incluirse controles internos positivos (estroma, linfocitos), que son importantes para poder determinar que la ausencia de expresión en un tumor no es debida a un problema de la técnica de inmunohistoquímica. Un caso se considera con pérdida de expresión cuando no se observa inmunotinción en ninguna célula neoplásica. Frecuentemente la tinción obtenida es heterogénea. Por ello, siempre se debe valorar la pérdida de expresión en un campo de gran aumento que disponga de controles internos positivos (Payá Romá et al., 2006; Perea et al., 2011).

\section{Definición de microsatélites y mecanismos de corrección}

Los microsatélites esta definidos como regiones del ADN con una unidad repetitiva de uno o más nucleótidos que son susceptibles a errores durante la replicación. Estos errores son corregidos por un sistema de reparación/correción. Sin embargo, cuando las proteínas reparadoras están mutadas los errores persisten. Estas proteínas reparadoras se expresan en los núcleos de las células colónicas normales y son detectables utilizando estudios de IHQ, mientras que los que presentan mutación no se expresan. Los genes MLH1, MSH2 pueden encontrarse mutados en el $90 \%$ de los casos de cáncer colorrectal y el resto corresponde a los genes MSH6 y PMS2. Estos estudios tienen alta sensibilidad y especificidad y son una opción válida para la detección del fenotipo mutador o síndrome de Lynch (Navarro et al., 2012; Ryan et al., 2017).

\section{Inestabilidad microsatelital}

El fallo para corregir errores en la replicación del ADN resulta en un fuerte fenotipo mutador. Las acumulaciones de alteraciones o errores conducen al desarrollo de inestabilidad microsatelital, conocido como fenotipo "inmune" debido a su alta antigenicidad (Ryan et al., 2017).

\section{Patogénesis del síndrome de Lynch}

El síndrome de Lynch se encuentra como de las más frecuentes neoplasias colorrectales hereditarias. Tiene su origen en mutaciones germinales que se pueden agrupar ya que los genes implicados codifican proteínas de reparación del ADN (MMR) (Cajal et al., 2016; Murillo Bacilio et al., 2018; Quezada Morales et al., 2018). Dichas proteínas del MMR, funcionan para mantener la fidelidad de la replicación del ADN previo a la mitosis y las diferencian de otros cánceres 
hereditarios como el CCR no polipósico hereditario y el síndrome de CCR familiar de tipo X y el síndrome de poliposis (Carethers, 2019).

La inestabilidad de microsatélites (IMS) corresponde a una vía molecular patogénica, como mutaciones puntuales, deleciones e inserciones, y se observa en aproximadamente el $15 \%$ de cánceres colorrectales. La inestabilidad de microsatélites alta o IMS-H por sus siglas en inglés se asocia a síndrome de Lynch en su mayoría, aunque existe casos esporádicos que se creen son causados por una inactivación epigenética del gen MLH1 (Gelsomino et al., 2016).

El síndrome de Lynch y los casos esporádicos de CCR presentan mutaciones que se dan principalmente en las secuencias microsatélites, distribuidas ampliamente en nuestro genoma, y genera una disminución o aumento del tamaño de los microsatélites en las células cancerosas. A este cambio en la longitud de los microsatélites se le denomina inestabilidad de microsatélites (IMS). Así, la IMS es una huella genética de la disfunción del sistema MMR. La IMS está presente en cáncer hereditario y esporádico, teniendo en ambos casos implicaciones clínicas para el paciente. En cáncer de tipo hereditario se presenta en pacientes con síndrome de Lynch cuya etiología se debe a mutaciones germinales en los genes del sistema MMR.

La IMS presenta la misma biología en casos hereditarios y esporádicos, por lo que el beneficio de conocer el estatus de IMS colaboraría en el tratamiento del paciente con CCR (Ortiz et al., 2016). Para el estudio de IMS recomendaron el uso del panel Bethesda, el cual consiste en el análisis de cinco marcadores microsatélites, tres de repeticiones dinucleotídicas: D2S123, D5S346 y D17S250; y dos de repeticiones mononucleotídicas: BAT25 y BAT26. Se estableció que dos o más de los marcadores propuestos deben encontrarse inestables para clasificar a un tumor con IMS-H, el cual tiene aplicación clínica demostrada. Si solo un marcador es inestable se clasifica como inestabilidad de microsatélites baja o IMSñL, cuyo significado biológico y clínico aún no ha sido dilucidado (Ortiz et al., 2016).

En una serie de casos, se determinó que el compromiso multi visceral de los tumores colónicos se presenta con una frecuencia de hasta $9.5 \%$ y que el tratamiento de elección es la resección R0, sin embargo, el obtener dichos resultados en una resección, se ve influido por el tipo histológico del tumor y su comportamiento biológico (Biel et al., 2017).

Además de la probabilidad de desarrollar CCR, la IMS se asocia al desarrollo de neoplasias de endo- metrio, intestino delgado, uréter o de la pelvis renal (Martínez et al., 2017a). En un caso reportado, se encontró sincrónicamente un CCR asociado a neoplasia de pulmón con mutación de receptor de factor de crecimiento epidérmico y un tumor neuroendocrino de apéndice cecal con IMS, esto de manera sincrónica, lo que ayudó a determinar que ambos tumores eran primarios y no metastásicos (Goyal et al., 2019).

Se ha observado que el involucramiento del colon izquierdo con inestabilidad alta de microsatélites se asocia con mayor frecuencia al síndrome de Lynch cuando se compara con IMSñH en lado derecho cuando no hay Lynch asociado, es decir, que cuando están ambos presentes, es más probable que ambos lados del colon estén afectados. En un estudio se observó una diferencia de asociación del $57 \%$ en lado izquierdo comparado con únicamente $20 \%$ en la afección derecha $(p=.008)$ (Hartman et al., 2013; Villegas-Mejía et al., 2018).

La población que presenta riesgo de desarrollar CCR esporádico son los individuos que se presentan asintomáticos de 50 años de edad o mayores que no tienen historia personal o familiar de CCR, pólipos adenomatosos o enfermedad inflamatoria intestinal. Estas características en una persona en riesgo, indica realizar estudios de tamizaje mediante prueba de sangre oculta en heces, sigmoidoscopia, enemas de Bario y colonoscopía cada 10 años, a diferencia de pacientes con sospecha de síndrome de Lynch en el que deben realizarse dichas pruebas anualmente (Heiken, 2001).

Debido a la edad más temprana de inicio, la colonoscopía debe de iniciarse desde los 25 años de edad y debido a su acelerada carcinogénesis, debe de repetirse cada año o cada dos años hasta los 40 años y luego anualmente. De ocurrir CCR, se debe de practicar una colectomía subtotal si así se requiriere. También debe de monitorizarse por posibilidad de cáncer sincrónico y metacrónico ( pueden aparecer tardíamente) en el endometrio, ovario, estómago, intestino delgado, páncreas, vías hepatobiliares y tracto urogenital superior (Lynch et al., 2009).

Por la alta prevalencia de CCR, identificar las vías diagnósticas y de tratamiento es de suma importancia. La IMS es un marcador significativo para la detección temprana de formas agresivas de la enfermedad. Las técnicas moleculares existen y proveen de un importante medio teranóstico (la palabra "teranóstico" se utiliza en patología para referirse a la aplicación de los resultados de inmunohistoquímica en el tratamiento del paciente, la palabra se utiliza en el título del libro 
de D. Dabbs: "Inmunohistoquímica diagnóstica, aplicaciones teranósticas y genómicas") al médico tratante (Nojadeh et al., 2018).

\section{Características Patológicas y Diagnóstico Inmunohistoquímico}

Las características histológicas pueden ser encontradas tanto en biopsias como en resecciones colónicas. Aunque estas características han sido incorporadas en los criterios revisados de Bethesda, no hay una única característica histológica que sea compatible con inestabilidad microsatelital, pero sí la puede predecir una combinación de ellas (Martínez et al., 2017b).

Aproximadamente del 15 al 20\% de los adenocarcinomas colorrectales surgen de deficiencias en la función del complejo MMR, lo que resulta en un alto nivel de IMS-H. Cuatro proteínas principales, MLH1, MSH2, MSH6 y PMS2, componen el complejo ADN MMR. Los carcinomas que salen de la vía IMS tienden a tener características patológicas particulares que incluyen una ubicación del lado derecho (cuando se encuentra IMS-H en ausencia de Síndrome de Lynch que suele presentarse en colon izquierdo), edad del paciente menor de 50 años, linfocitos infiltrantes de tumor, una falta de "necrosis sucia", presencia de agregados linfoides peritumorales (Reacción tipo-Crohn), diferenciación mucinoso, características medulares y una neoplasia que está bien diferenciada (Yilmaz et al., 2019; Yuan et al., 2015).

En adenocarcinoma IMS-H esporádicos no hereditarios, la hipermetilación del promotor del gen $M L H 1$ conduce a deficiencias en la expresión de la proteína MLH1, lo cual origina ausencia de tinción de la proteína nuclear en células tumorales. En los adenocarcinomas hereditarios (síndrome de Lynch), las mutaciones en la línea germinal afectan más comúnmente el gen MSH2, así como a los genes MLH1, MSH6 y PMS2, lo cual causa pérdida de tinción nuclear de la proteína particular (Vaccaro et al., 2007). Los anticuerpos contra MLH1, MSH2, MSH6 y PMS2 se utilizan para detectar tanto el síndrome de Lynch, IMS esporádica e IMS-H con implicaciones terapéuticas y pronósticas. $\mathrm{Al}$ interpretar estas tinciones, es importante observar que MLH1 existe como un heterodímero con PMS2 y MSH2 y se heterodimeriza con MSH6.

Por lo tanto, si hay un defecto de MLH1, también se detectará la pérdida de PMS2. Sin embargo, si la expresión de la proteína PMS2 está comprometida, el MLH1 permanecerá intacto. El par MSH2/MSH6 muestra un patrón similar de reactividad, un defecto del gen MSH6 causa solo pérdida de MSH6, pero el compromiso de $\mathrm{MSH} 2$ produce la pérdida tanto de MSH2 como de MSH6. Por esta razón es que algunos autores han propuesto restringir el panel de cuatro anticuerpos a uno de dos anticuerpos PMS2 y MSH6 (Cheah et al., 2019; Geiersbach \& Samowitz, 2011; Jung et al., 2017; Kumarasinghe et al., 2010; Schmitz et al., 2014; Shia, 2008).

Un intento de unificar las diferentes expresiones de los anticuerpos para MSH1, MSH2, MSH6 y PMS2 concluyó que hay interconectividad entre seis clasificaciones independientes que se resumen en cuatro subtipos moleculares que distinguen entre hipermutación e inestabilidad de microsatélites, mutación epitelial cromosómica inestable y desregulación metabólica (Lee et al., 2019; Zakout \& Lanza, 2019; Zhang \& Li, 2013).

Cada vía molecular que expresa el CCR se resume en dos: una vía supresora y una vía mutadora. La vía supresora la acompaña una inestabilidad cromosómica que conduce a mutaciones varias. Dicha vía la conforman en un $85 \%$ los tumores esporádicos. La vía mutadora se caracteriza por la inestabilidad de microsatélites (Caldés Llopis, 2003).

Los síndromes heredados como la poliposis adenomatosa familiar y el síndrome de Lynch tienen un perfil molecular característico. En general, los cánceres se dividen en dos grupos generales basados en su diferenciación genómica: la inestabilidad cromosómica y la inestabilidad microsatelital (Gibson et al., 2014; Marisa et al., 2013).

\section{IMS como factor predictivo de la eficacia de la inmunoterapia anti PD1/PD-L1 en CCR}

Recientemente se ha considerado la posibilidad de tratar pacientes con estrategias de inmunoterapia utilizando bloqueadores de PD-1MAb (Pembrolizumab). Se han realizado estudios en los cuales se refuerza la hipótesis de que los tumores que presentan inestabilidad microsatelital tienen una mayor respuesta al bloqueo de PD-L1, ya que dichos tumores tienen alterado el sistema MMR ya que la pérdida de las proteínas de MMR se ha correlacionado con expresión tumoral de PD-L1. Pacientes con IMS y otros tumores asociados a IMS han tenido un alto porcentaje de respuesta hasta de $40 \%$ y $71 \%$ en algunos casos (Zhao et al., 2019).

La inmunoterapia ha demostrado resultados prometedores en varios tipos de cáncer, sin embargo, 
como se ha mencionado, el Pembrolizumab ha sido aprobado para tratamiento basado en un biomarcador, el IMS-H. Se considera que dicha alteración provoca un acúmulo de mutaciones somáticas y que dicho espectro de mutaciones provoca una carga elevada de expresiones antigénicas y una subsecuente acumulación de linfocitos que infiltran el tumor. Estos cambios se han asociado a la aumentada sensibilidad de medicamentos como Pembrolizumab (Chang et al., 2017).

Los estudios de IHQ como el análisis de microsatélites, son técnicas igualmente válidas para el estudio de tumores con fenotipo mutador y para el diagnóstico de pacientes con síndrome de Lynch. La IHQ como método inicial tiene la ventaja de que es una técnica sencilla y barata y accesible. Es fácilmente realizable en cualquier laboratorio que rutinariamente realicen tinciones inmunohistoquímicas en sus laboratorios de Patología Anatómica. El análisis de microsatélites requiere un mayor equipamiento técnico, no disponible en todos los laboratorios. Adicional a esto, la inmunohistoquímica permite identificar la proteína no expresada y, por tanto, el gen afectado. También se ha visto que en tumores mucinosos tienen mayor sensibilidad y se detectan mejor por inmunohistoquímica que por el análisis de microsatélites, debido a la baja densidad de células tumorales, que dificulta la obtención de suficiente ADN para el estudio (Payá Romá et al., 2006). Sin embargo existe un consenso interinstitucional de realizar estudios de tamizaje universal para CCR tanto por métodos de patología molecular como por IHQ para MMR con la finalidad de verificar la presencia de fenotipo de inestabilidad (Ryan et al., 2017).

La IHQ toma especial importancia ya que existen estudios que sugieren que las terapias de combinación de inhibidores de puntos de verificación como anti-PD-L1, puede conllevar un mejor pronóstico cuando los pacientes tiene estudiado el fenotipo MMRIMS-H en CCR. Se han iniciado estudios con la combinación de Durvalumab y Tremelumumab que son anti PD-L1 y anti CTLA-4 respectivamente (Kreidieh et al., 2020).

Adicionalmente a los estudios de IHQ se suma la importancia de los estudios de patología molecular, que evalúan el estatus de inestabilidad de microsatélites junto con los estudios de las mutaciones de $N R A S$, $K R A S$ y $B R A F$. La asociación de IMS con metilación del promotor $M L H 1$ en conjunto con la activación de la mutación $B R A F$, puede ofrecer criterios de exclusión para pruebas genéticas posteriores, mientras que la au- sencia deéstas puede sugerir la presencia de síndrome de Lynch. Además, con la información del tamizaje ahora se puede determinar el pronóstico e implicaciones terapéuticas de los pacientes (Harada \& Morlote 2020; Ryan et al., 2017).

Si bien ya se observa una respuesta positiva tras la selección de candidatos para tratamiento con antiPD-L1 mediante técnicas de IHQ y de pruebas moleculares, también los estudios mencionados han sido de gran utilidad para combinar los tratamientos antiPD-L1 con medicamentos antiñangiogénicos que pueden ser utilizados en conjunto especialmente al estudiar el microambiente del tumor (Fridman et al., 2020).

\section{Conclusión}

El CCR es uno de los cánceres más comunes en el mundo y del 15 al 20\% de estos tumores tienen el fenotipo de inestabilidad microsatelital que muestra una deficiencia en las proteínas reparadoras del ADN. La inestabilidad microsatelital es una de las características del síndrome de Lynch, sin embargo, existe un número elevado de tumores con inestabilidad microsatelital que ocurren esporádicamente como resultado de una mutación adquirida en genes que codifican las proteínas reparadoras del ADN. La identificación de inestabilidad microsatelital puede ser realizada en forma indirecta por la detección de la expresión de las proteínas reparadoras del ADN: MLH1, MSH2, MSH6 y PMS2 mediante IHQ, las cuales no se expresan si se encuentra presente el fenotipo de inestabilidad microsatelital.

La identificación de pacientes con CCR con inestabilidad microsatelital, tiene importantes implicaciones clínicas tales como un marcador predictivo para quimioterapia, para utilizar agentes de inmunoterapia (bloqueador de PD-1) y como parte del algoritmo en el diagnóstico del síndrome de Lynch. Esto ayuda a personalizar la terapia en pacientes con cáncer de colon con una mejor estrategia en su seguimiento y tratamiento. En Guatemala, es necesario conocer el porcentaje de casos con inmunofenotipo de inestabilidad microsatelital en CCR mediante estudios de investigación ya que es necesario implementar un tamizaje para IMS en CCR tanto para generar estadísticas sobre el fenotipo de la población guatemalteca y para encontrar candidatos para la terapia dirigida con agentes de inmunoterapia. 


\section{Contribución de los autores}

Coordinación, elaboración y revisión del Documento: Todos los autores

\section{Materiales suplementarios}

Los materiales suplementarios de este artículo se encuentran en la página web de la revista a través del doi: https://doi.org/10.36829/63CTS.v8i2.945

\section{Referencias}

Adar, T., Rodgers, L. H., Shannon, K. M., Yoshida, M., Ma, T., Mattia, A., Lauwers, G. Y., Iafrate, A. J., \& Chung, D. C. (2017). A tailored approach to BRAF and MLH1 methylation testing in a universal screening program for Lynch syndrome. Modern Pathology, 30(3), 440-447. https://doi.org/10.1038/modpathol.2016.211

Alhilfi, H. S. Q., Almohammadawi, K. O. M., Alsaad, R. K. A., Ameen, N. A., Aliedani, B. K. A., Aldubaisi, H. J. I., \& Alshewered, A. S. H. (2019). Colorectal cancer epidemiology and clinical study in Misan. Journal of Coloproctology, 39(2), 159-162. https://doi.org/10.1016/j.jcol.2018.12.001

Battaglin, F., Naseem, M., Lenz, H. J., \& Salem, M. E. (2018). Microsatellite instability in colorectal cancer: Overview of its clinical significance and novel perspectives. Clinical Advances in Hematology and Oncology, 16(11), 735-747.

Biel, W. E., Walker, G. G., Ocares, U. M., Benavides, Y. C., Martin, Q. F., Vivanco, A. J., Zuniga, T. C., Jerez, S. J., Lara, A. E., \& Caselli, M. G. (2017). Early and late results after multivisceral resections for T4b colonic cancer TT - Resultados precoces y tardios en resecciones ampliadas en cancer de colon T4b. Revista Chilena de Cirugia, 69(2), 139-143. https://doi.org/http://dx.doi.org/ 10.1016/j.rchic.2016.10.006

Burbano Luna, D. F., Manrique, M. A., Chávez García, M. ç., Pérez Corona, T., Hernández Velázquez, N. N., Escandón Espinoza, Y. M., Gómez Urrutia, J. M., Rubalcaba Macías, E. J., Martínez Ramírez, G., Cisneros, A. A., García Romero, A., Martínez
Galindo, M. G., \& Cerna Cardona, J. (2016). Epidemiología del cáncer colorrectal en menores de 50 años en el Hospital Juárez de México. Endoscopia, 28(4), 160-165. https://doi.org/ 10.1016/j.endomx.2016.10.008

Cajal, A., Piñero, T., Verzura, A., Santino, J., Solano, A., Kalfayan, P., Ferro, F., \& Vaccaro, C. (2016). Mutación fundadora en síndrome de Lynch tipo II. Medicina (Buenos Aires), 76(1), 180-182.

Caldés Llopis, T. (2003). Diagnóstico Molecular del Cáncer Colorrectal Hereditario. Seom, 1(1), 184 ñ189.

Carethers, J. M. (2019). High predictability for identifying Lynch syndrome via microsatellite instability testing or immunohistochemistry in all Lynch-associated tumor types. Translational Cancer Research, 8(Suppl 6), S559-S563. https://doi.org/10.21037/tcr.2019.08.10

Castells, A., Castellví-Bel, S., \& Balaguer, F. (2009). Concepts in Familial Colorectal Cancer: Where Do We Stand and What Is the Future? Gastroenterology, 137(2), 404-409. https://doi.org/ 10.1053/j.gastro.2009.06.015

Chang, L., Chang, M., Chang, H. M., \& Chang, F. (2017). Microsatellite Instability. Applied Immunohistochemistry \& Molecular Morphology, 26(2), 1. https://doi.org/10.1097/PAI. 0000000000000575

Cheah, P. L., Li, J., Looi, L. M., Koh, C. C., Lau, T. P., Chang, S. W., Teoh, K. H., Mun, K. S., \& Nazarina, A. R. (2019). Screening for microsatellite instability in colorectal carcinoma: Practical utility of immunohistochemistry and pcr with fragment analysis in a diagnostic histopathology setting. Malaysian Journal of Pathology, 41(2), 91-100.

Deen, K. I., Silva, H., Deen, R., \& Chandrasinghe, P. C. (2016). Colorectal cancer in the young, many questions, few answers. World Journal of Gastrointestinal Oncology, 8(6), 481. https://doi.org/10.4251/wjgo.v8.i6.481

Fridman, W. H., Miller, I., Saut s-Fridman, C., \& Byrne, A. T. (2020). Therapeutic Targeting of the Colorectal Tumor Stroma. Gastroenterology, 158(2), 303-321. https://doi.org/10.1053/j. gastro.2019.09.045 
Geiersbach, K. B., \& Samowitz, W. S. (2011). Microsatellite instability and colorectal cancer. Archives of Pathology and Laboratory Medicine, 135(10), 1269-1277. https://doi.org/10.5858/ arpa.2011-0035-RA

Gelsomino, F., Barbolini, M., Spallanzani, A., Pugliese, G., \& Cascinu, S. (2016). The evolving role of microsatellite instability in colorectal cancer: A review. Cancer Treatment Reviews, 51, 19-26. https://doi.org/10.1016/j.ctrv.2016.10.005

Giardiello, F. M., Allen, J. I., Axilbund, J. E., Boland, C. R., Burke, C. A., Burt, R. W., Church, J. M., Dominitz, J. A., Johnson, D. A., Kaltenbach, T., Levin, T. R., Lieberman, D. A., Robertson, D. J., Syngal, S., \& Rex, D. K. (2014). Guidelines on genetic evaluation and management of lynch syndrome: A consensus statement by the us multiñsociety task force on colorectal cancer. Gastroenterology, 147(2), 502-526. https://doi.org/ 10.1053/j.gastro.2014.04.001

Gibson, J., Lacy, J., Matloff, E., \& Robert, M. (2014). Microsatellite instability testing in colorectal carcinoma: A practical guide. Clinical Gastroenterology and Hepatology, 12(2), 171-176. e1. https://doi.org/10.1016/j.cgh.2013.11.001

Goyal, S., Botra, S., Sharma, M., Gupta, M., Koyyala, V. B., Jajodia, A., \& Sranagi, P. (2019). Sporadic synchronous triple primary cancers in elderly female: Microsatellite instability high resectable colon cancer, epidermal growth factor receptor-mutated metastatic lung cancer, and neuroendocrine tumor of appendix. Oncology Journal of India, 3(2), 45. https://doi.org/10.4103/ oji.oji_19_19

Hartman, D. J., Brand, R. E., Hu, H., Bahary, N., Dudley, B., Chiosea, S. I., Nikiforova, M. N., \& Pai, R. K. (2013). Lynch syndromeñassociated colorectal carcinoma: Frequent involvement of the left colon and rectum and lateñonset presentation supports a universal screening approach. Human Pathology, 44(11), 2518-2528. https://doi.org/10.1016/j.humpath.2013.06.012

Heiken, J. P. (2001). Colon cancer screening. Cancer Imaging, 2(1), 10-14. https://doi.org/10.1102/14707330.2001 .008

Jung, J., Kang, Y., Lee, Y. J., Kim, E., Ahn, B., Lee, E., Kim, J. Y., Lee, J. H., Lee, Y., Kim, C. H., \&
Chae, Y. S. (2017). Comparison of the mismatch repair system between primary and metastatic colorectal cancers using immunohistochemistry. Journal of Pathology and Translational Medicine, 51(2), 129-136. https://doi.org/10.4132/ jptm.2016.12.09

Kreidieh, M., Mukherji, D., Temraz, S., \& Shamseddine, A. (2020). Expanding the Scope of Immunotherapy in Colorectal Cancer: Current Clinical Approaches and Future Directions. BioMed Research International, 2020. https://doi.org/ 10.1155/2020/9037217

Kumarasinghe, A. P., De Boer, B., Bateman, A. C., \& Kumarasinghe, M. P. (2010). DNA mismatch repair enzyme immunohistochemistry in colorectal cancer: A comparison of biopsy and resection material. Pathology, 42(5), 414-420. https://doi.org/10.3109/00313025.2010.493862

Lee, M. J., Kwon, J. S., Jiang, H. B., Choi, E. H., Park, G., \& Kim, K. M. (2019). The antibacterial effect of non-thermal atmospheric pressure plasma treatment of titanium surfaces according to the bacterial wall structure. Scientific Reports, 9(1), 1-13. https://doi.org/10.1038/s41598-019-39414-9

Lynch, H. T., Lynch, P. M., Lanspa, S. J., Snyder, C. L., Lynch, J. F., \& Boland, C. R. (2009). Review of the Lynch syndrome: History, molecular genetics, screening, differential diagnosis, and medicolegal ramifications. Clinical Genetics, 76(1), 1-18. https://doi.org/10.1111/j.1399-0004.2009.01230.x

Marisa, L., de Reyni s, A., Duval, A., Selves, J., Gaub, M. P., Vescovo, L., Etienne-Grimaldi, M. C., Schiappa, R., Guenot, D., Ayadi, M., Kirzin, S., Chazal, M., Fléjou, J. F., Benchimol, D., Berger, A., Lagarde, A., Pencreach, E., Piard, F., Elias, D., É Boige, V. (2013). Gene Expression Classification of Colon Cancer into Molecular Subtypes: Characterization, Validation, and Prognostic Value. PLoS Medicine, 10(5). https://doi.org/10.1371/journal.pmed.1001453

Martínez, C. I., Pérez, L. F., Baquero, D., \& Barco, A. (2017a). Cáncer colorrectal hereditario no asociado a poliposis o síndrome de Lynch. Revista Colombiana de Cirugía, 32(4), 297-303. https://doi.org/10.30944/20117582.38

Martínez, C. I., Pérez, L. F., Baquero, D., \& Barco, A. (2017b). Cáncer colorrectal hereditario no 
asociado a poliposis o síndrome de Lynch. Revista Colombiana de Cirugía, 32(4), 297-303. https://doi.org/10.30944/20117582.38

Murillo Bacilio, M., Palta Gonzalez, A., \& Correa Martínez, F. (2018). Inestabilidad de los microsatélites en cáncer colo-rectal y su distribución de acuerdo a factores pronósticos en SOLCA Cuenca 2004-2014. Revista de La Facultad de Ciencias Médicas Universidad de Cuenca, 36(1), 9-16.

Navarro, S., Pérez-Segura, P., Ramón y Cajal, S., Salazar, R., García-Foncillas, J., Musulén Palet, E., García-Carbonero, R., Payá, A., \& García-Alfonso, P. (2012). Recomendación para la determinación de biomarcadores en el carcinoma colorrectal. Consenso Nacional de la Sociedad Española de Anatomía Patológica y de la Sociedad Española de Oncología Médica. Revista Espanola de Patologia, 45(3), 130-144. https://doi.org/10.1016/j.patol.2012.05.001

Nojadeh, J. N., Sharif, S. B., \& Sakhinia, E. (2018). Microsatellite instability in colorectal cancer. EXCLI Journal, 17, 159-168. https://doi.org/ 10.17179/excli2017-948

Ortiz, C., Dongo-Pflucker, K., Martín-Cruz, L., Barletta Carrillo, C., Mora-Alferez, P., \& Arias, A. (2016). Microsatellite instability in patients with diagnostic of colorectal cancer. Revista de Gastroenterologia Del Peru : Organo Oficial de La Sociedad de Gastroenterologia Del Peru, 36(1), 15-22.

Payá Romá, A., Alenda González, C., Jover Martínez, R., \& Aranda López, F. I. (2006). Carcinoma colorrectal con alteración de la vía reparadora. Claves para su identificación y relevancia clínica. Revista Española de Patología, 39(4), 201-208. https://doi.org/10.1016/s1699-8855(06)70042-3

Perea, J., Lomas, M., \& Hidalgo, M. (2011). Bases moleculares del cáncer colorrectal: ¿Hacia un manejo individualizado? Revista Espanola de Enfermedades Digestivas, 103(1), 29-35. https://doi.org/10.4321/S1130-01082011000100006

Quezada Morales, M. E., Guallasamin Chalco, E. F., Jara Sanchez, H. E., \& Fajardo Morales, P. F. (2018). Síndrome de Lynch: Caracterización genético clínica y epidemiología. Caso clínico TT - Lynch syndrome: clinical genetic characterization and epidemiology. Clinical case. Rev. Fac. Cienc. Méd. Univ. Cuenca, 36(1), 61-68. https://publicaciones. ucuenca.edu.ec/ojs/index.php/medicina/article/ view/2085/1497

Ryan, E., Sheahan, K., Creavin, B., Mohan, H. M., $\&$ Winter, D. C. (2017). The current value of determining the mismatch repair status of colorectal cancer: A rationale for routine testing. Critical Reviews in Oncology/Hematology, 116, 38-57. https://doi.org/10.1016/j.critrevonc. 2017.05.006

Schmitz, L., Moretti, L., Marino, L., Gimenez, L., \& Rojas Bilbao, E. (2014). Estudio fenotípico de inestabilidad microsatelital en cáncer colorrectal. Correlación con parámetros histológicos y clínicos. Revista Española de Patología, 47(4), 204-209. https://doi.org/10.1016/j.patol. 2013.10.002

Shia, J. (2008). Immunohistochemistry versus microsatellite instability testing for screening colorectal cancer patients at risk for hereditary nonpolyposis colorectal cancer syndrome: Part I. The utility of immunohistochemistry. Journal of Molecular Diagnostics, 10(4), 293-300. https://doi.org/10.2353/jmoldx.2008.080031

Vaccaro, C. A., Carrozzo, J. E., Mocetti, E., Berho, M., Valdemoros, P., Mullen, E., Oviedo, M., \& Redal, M. A. (2007). Expresion inmunohistoquimica e inestabilidad microsatelital en el sindrome de lynch. Medicina, 67(3), 274-278.

Vasen, H. F. A., Blanco, I., Aktan-Collan, K., Gopie, J. P., Alonso, A., Aretz, S., Bernstein, I., Bertario, L., Burn, J., Capella, G., Colas, C., Engel, C., Frayling, I. M., Genuardi, M., Heinimann, K., Hes, F. J., Hodgson, S. V., Karagiannis, J. A., Lalloo, F., É Mšslein, G. (2013). Revised guidelines for the clinical management of Lynch syndrome (HNPCC): recommendations by a group of European experts. Gut, 62(6), 812-823. https://doi.org/10.1136/gutjnl-2012-304356

Villegas-Mejía, C. R., Chacón-Cardona, J. A., Villegas-Jaramillo, M., \& Villegas-Jaramillo, P. (2018). Cáncer de colon Àuna sola entidad? Consideraciones sobre lateralidad en una institución oncológica colombiana 2001-2016. Ces Medicina, 32(3), 191-202. https://doi.org/ 10.21615/cesmedicina.32.3.1 
Yilmaz, R., Bedir, R., Akdogan, R. A., \& Pergel, A. (2019). Evaluation of Microsatellite Instability in Colorectal Adenomas and Carcinomas by Immunohistochemistry and a Comparison of Histopathological Features. European Journal of Therapeutics, 25(1), 28-28. https://doi.org/ 10.5152/eurjther.2018.582

Yuan, L., Chi, Y., Chen, W., Chen, X., Wei, P., Sheng, W., Zhou, X., \& Shi, D. (2015). Immunohistochemistry and microsatellite instability analysis in molecular subtyping of colorectal carcinoma based on mismatch repair competency. International Journal of Clinical and Experimental Medicine, 8(11), 20988-21000.
Zakout, Y. M. A., \& Lanza, G. (2019). Detection of DNA Mismatch Repair Protein Abnormalities in Sudanese Colorectal Cancer Patients Using Immunohistochemical Methods. Journal of Gastrointestinal Cancer, 50(3), 530-536. https://doi.org/10.1007/s12029-018-0118-z

Zhang, X., \& Li, J. (2013). Era of universal testing of microsatellite instability in colorectal cancer. World Journal of Gastrointestinal Oncology, 5(2), 12. https://doi.org/10.4251/wjgo.v5.i2.12

Zhao, P., Li, L., Jiang, X., \& Li, Q. (2019). Mismatch repair deficiency/microsatellite instability-high as a predictor for anti-PD-1/PD-L1 immunotherapy efficacy. Journal of Hematology and Oncology, 12(1), 1-14. https://doi.org/10.1186/s13045-0190738-1 